\title{
A Wireless Health Monitoring System
}

\author{
Edward Teaw, Guofeng Hou, \\ Michael Gouzman, K. Wendy Tang \\ Department of Electrical and Computer Engineering \\ Stony Brook University \\ Stony Brook, New York 11994, USA \\ \{wtang,mig,guhou\}@ece.sunysb.edu \\ Matthew Kane \\ Department of Mechanical Engineering \\ Farmingdale State University \\ Farmingdale, NY 11735, USA
}

\author{
Amy Kesluk \\ Computer Science Department \\ Hofstra University \\ Hemstead, New York 11549, USA
}

\author{
Jason Farrell \\ Engineering Division \\ Suffolk Community College \\ Selden, NY 11784, USA
}

\begin{abstract}
With the aging of the baby boomers, it is predicted that the US population over age 65 will grow from its 1999 level of 34.6 million persons to approximately 82 million in 2050 , a $137 \%$ increase. The most rapid surge in our senior population will take place between 2011 and 2030. During this 19-year interval, seniors will expand from $13 \%$ of our population to $22 \%$ of our population.
\end{abstract}

In this project, our goal is to design a wireless sensor system, the Health Tracker 2000, that can monitors users' vital signs and notifies relatives and medical personnel of their location during life threatening situations.

The Health Tracker 2000 combines wireless sensor networks, existing RFID (Radio Frequency Identification) and Vital Sign Monitoring technology to simultaneously monitor vital signs while keeping track of the users' location. The use of wireless technology makes it possible to install the system in all types of homes and facilities. Radio frequency waves can travel through walls and fabric, sending the vital sign and location information to a central monitoring computer via a miniature transmitter network. Such information can easily be accessed from any location over the Internet.

Index Terms - sensor networks, vital sign sensors.

\section{INTRODUCTION}

The speed of change in the medical field has been overwhelming. Groundbreaking achievements such as the discovery and development of penicillin, chemotherapy, and vaccinations have led people in the medical profession to have a great understanding of the human body [1]. The average life expectancy in the United States has increased from 47.3 years in 1900 to 68.2 years in 1950 to 77.3 years in 2002 [2,3]. With such a high and continued increasing average life expectancy rate, medical care for senior citizens, age 65 and over, is becoming progressively more important.
The evolution of wireless technology is also extremely fast-paced. The $802.11 \mathrm{~b}$ protocol for wireless computer networks came in large demand in 2000. In just over four years, wireless communications technology has become readily available for the general public, with 7.5 million households in the U.S. using some form of a wireless network [4]. The benefits of wireless technology are already apparent: portability, convenience, ease of installation, and low cost.

What if wireless and medical sensor technology were combined? In this paper, we discuss the design of a wearable device that can remotely monitor vital signs of users. This device is implemented using existing technologies. The information from this device is sent to a base station which is connected to a computer. The information will be received by medical personnel and/or family members. Several patients may be monitored from a single base station. The system is designed so that it is easy to use and set up in medical facilities (such as hospitals) and residences. Figure 1 is an overview of the Wireless Health Monitoring system.

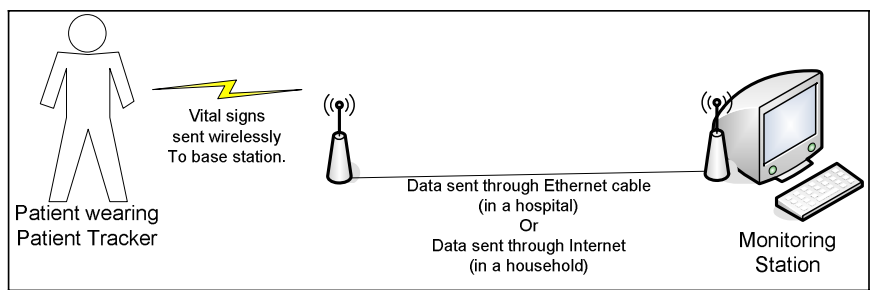

Fig. 1: System Overview 


\section{SYSTEM DESIGN}

\section{A. Overall System Design}

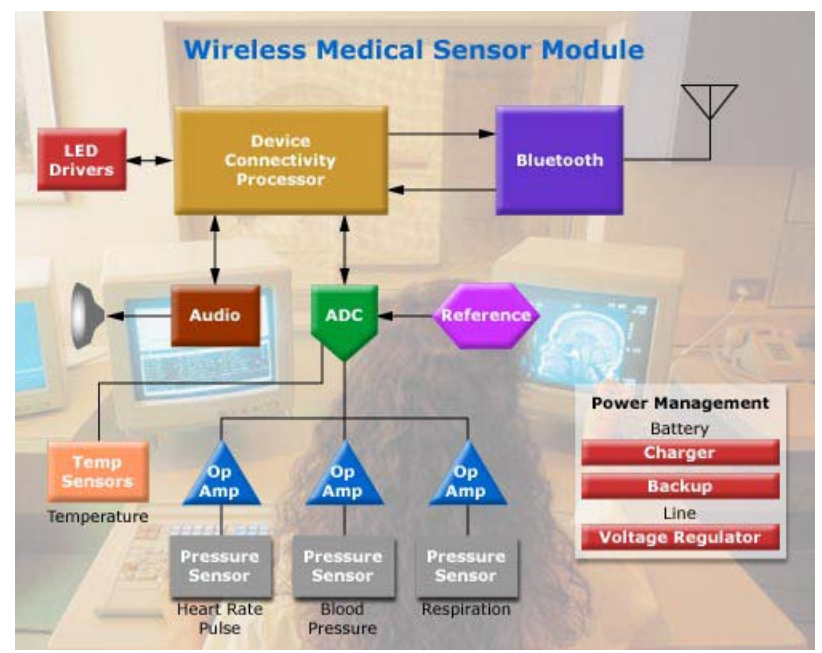

Fig 2: Wireless Medical Sensor Module

Figure 2 shows the general layout of a wireless medical sensor [5]. The vital signs monitored shown in the diagram are heart rate/pulse, blood pressure, respiration rate, and body temperature. The sensors for heart rate/pulse, blood pressure, and respiration rate could be implemented using pressure sensors. To ensure a proper reading of these sensor outputs, the signals must be amplified using op amps. The outputs from the temperature sensor and all three op amps are converted to a digital signal using an ADC (analog to digital converter). These signals are processed using a microcontroller or microprocessor and the data is output via a wireless module.
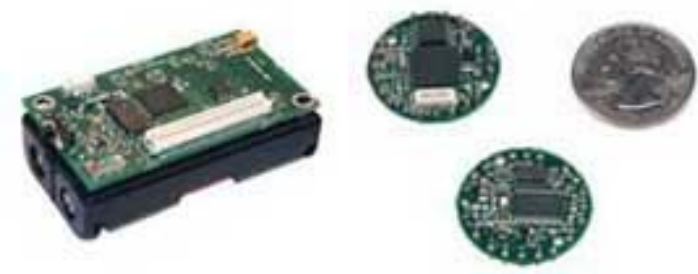

Fig. 3: Crossbow MICA2 (left) and MICA2DOT (right)

\section{B. Wireless Transceivers and Receivers}

The wireless transceivers selected, the Crossbow MICA2 and MICA2DOT motes (Fig 3), have embedded within a wireless transmitter/receiver, microcontroller, and an ADC [6]. These motes transmit data using RF communication, with the ability to transmit from $433 \mathrm{MHz}$ to $900 \mathrm{MHz}$. The MICA2 and MICA2DOT motes can work together in a mesh network (Fig 4). Each of these motes can work as either a node in the mesh network or a base station when plugged into an interface board which is connected to a computer via a serial cable (Figure 4).

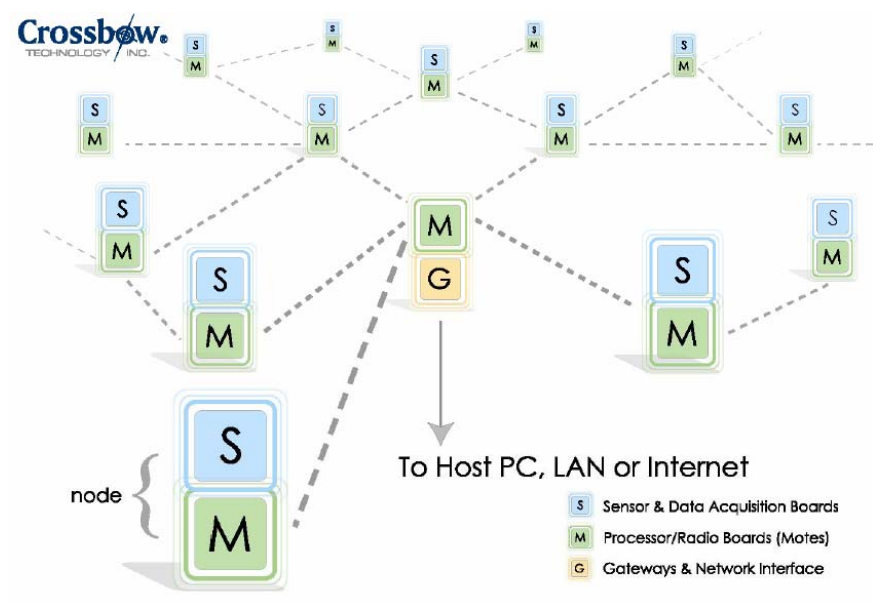

Fig. 4: Mesh Network Topology

Since a mesh network topology is used, the range of the whole network could be extended by hoping through multiple motes. Another advantage of a multi-hop mesh network topology is that if a node becomes faulty, say for example its battery died, communications of the network can still be maintained by routing through alternate routs.

The MICA2 has a 51-pin connector (Fig 5). This mote is connected to various vital sign sensors via sensor acquisition boards, MDA310 and MDA510, also provided by Crossbow Technologies Inc. More specifically, the MDA310 is compatible with the MICA2 and has seven ADC inputs for up to seven sensors. The MDA510 is the sensor acquisition board compatible with the MICA2DOT. With this board we have the ability to connect up to five sensors through the ADC inputs.

\section{Sensors}

The wireless medical sensor we are designing measures several vital signs of the user. Specifically, these vital signs are: temperature, pulse rate, breath rate, and blood oxygen level.

\section{Thermal}

In selecting thermal sensors, we first considered the National Semiconductor LM34 Precision Fahrenheit Temperature Sensor. There are several problems in using the LM34. The first problem is the supply voltage. The LM34 requires at least $5 \mathrm{~V}$ at the input. This requires us to add extra batteries and a voltage regulator which means extra components and larger size for the final product. The second problem is that the LM34 has a resolution of $1{ }^{\circ} \mathrm{F}$. This is too high for medical applications because since body temperature of a healthy human is $98.6^{\circ} \mathrm{F}$, the LM34 will accurately read this as either $98^{\circ} \mathrm{F}$ or $99^{\circ} \mathrm{F}$. 


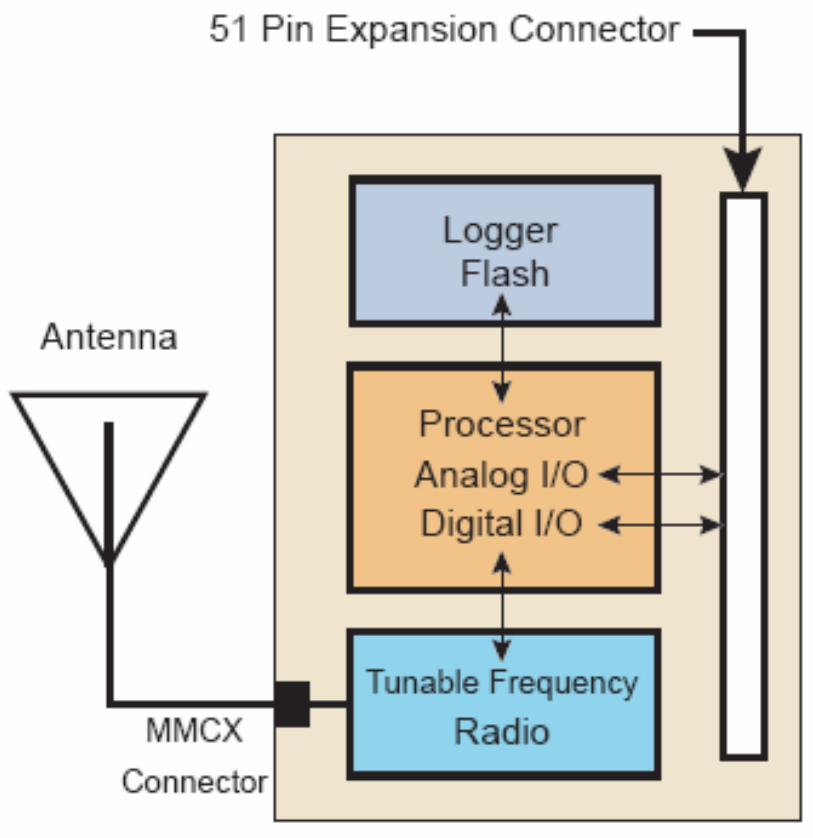

Fig. 5: Block Diagram of a MICA2

We then consider the LM92 by National Semiconductors. The major difference is that the LM92 has a serial digital output (Fig 6). Since the MDA510 does not have any digital inputs or outputs, we must use the LM92 with the MDA310 which is compatible with the MICA2. The LM92 has a minimum supply voltage of $2.7 \mathrm{~V}$ and a resolution of $0.3^{\circ} \mathrm{F}$. This component is clearly the ideal choice for our system.

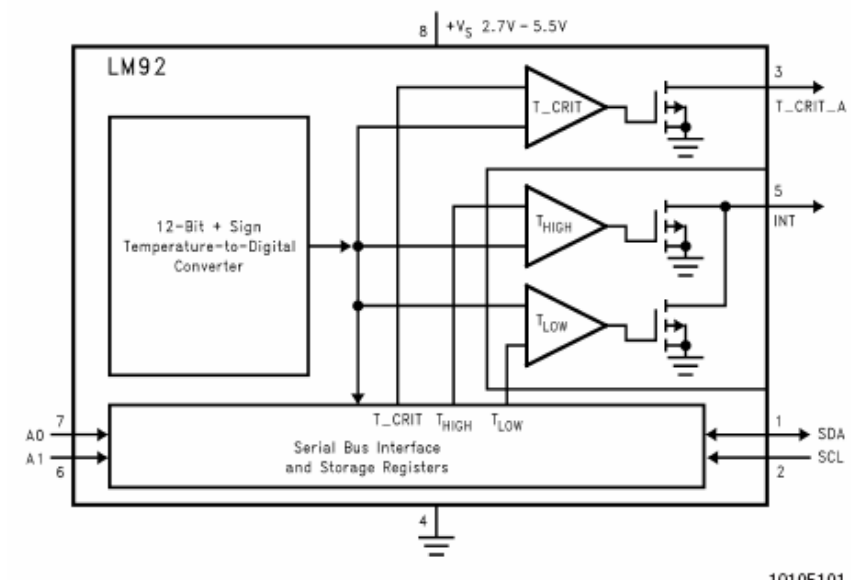

Fig 6: Schematic of LM92

An additional advantage in choosing the LM92 is that since it has a $2.7 \mathrm{~V}$ minimum supply voltage, we are able to use the INT outputs on the MICA2. The INT outputs supply power to the sensors only when the mote is polling for data. Since the LM34 was connected to the $5 \mathrm{~V}$ voltage regulator which is connected directly to the battery, it is always draining the battery. The INT outputs generate a $3 \mathrm{~V}$ square wave (Fig
7) that is asserted when obtaining sensor data and unasserted when the mote is in sleep mode. This will considerably extend the battery life when used in comparison with the LM34.

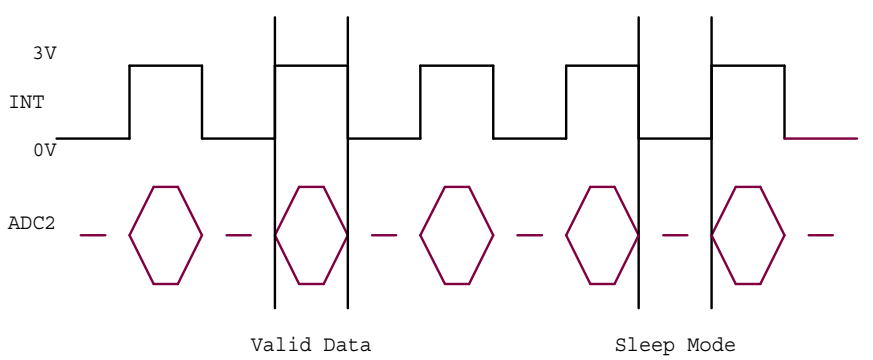

Fig. 7: Timing Diagram for Temperature Sensor

\section{$\underline{\text { Oximeter }}$}

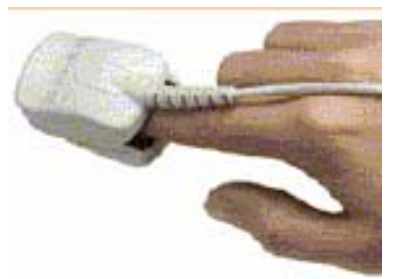

Fig 8: Nonin ipod

The Nonin ipod (Fig 8) is a digital oximeter and sensor that goes over the user's finger [7]. The unit itself is simply a module that can be readily integrated into a host device. The module measures the blood oxygen level and pulse rate. The oximeter and sensor is contained in a single unit, saving space in the final product. The ipod will be integrated into the system via a 3-wire interface with serial communications, similar to that of the LM92 temperature sensor. Since it is a digital output the unit will interface with the MICA2 via the digital inputs. The ipod has a minimum operating voltage of $2 \mathrm{~V}$ with a maximum of $6 \mathrm{~V}$, thus matching the specifications of our supply voltage of $3 \mathrm{~V}$.

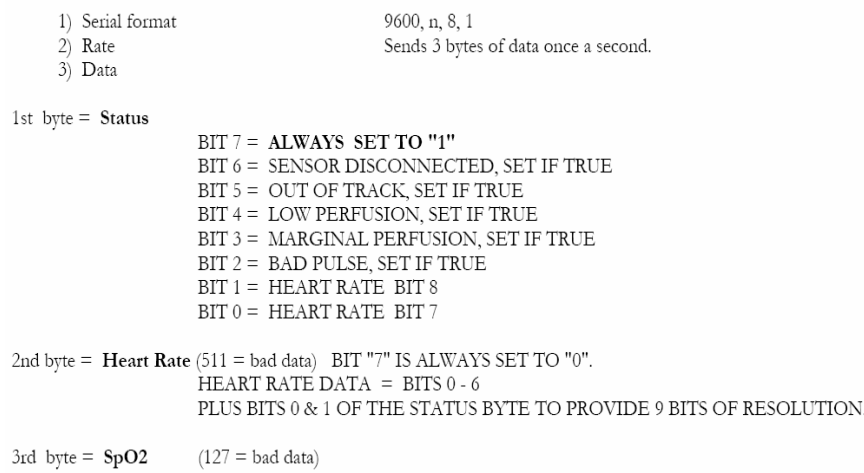

Fig 9: Format of Nonin ipod output 
The ipod has a serial digital output. Fig 9 shows the format of the output. The data from the device is sent at a rate of 3 bytes per second. The first byte is the status byte, the second is the heart rate, and the third is the blood oximetry. Some experimentation is needed to correctly interpret the data sent to the mote. With serial data transmissions timing is always an issue. Normally a serial device transmits data by latching each bit by means of a clock signal and a data signal.

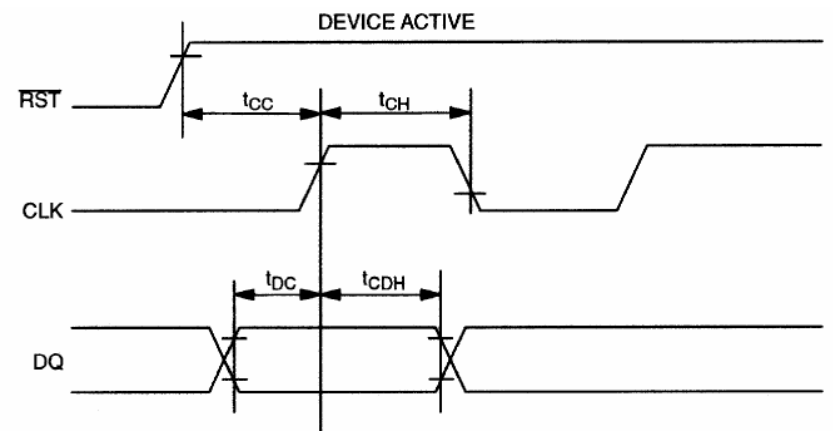

Fig. 10: Serial Data Transfer timing waveform

Fig 10 shows a timing waveform for a typical serial data device. The /RST bit is unasserted to allow data to transfer. The DQ sends the first bit and the bit is stored when CLK goes high. The timing waveform for the device above is an active high latch, since the data is being sent when the CLK bit is high and not during the positive or negative edge. In other words, bit DQ is sent only when the CLK bit is in the tCH region shown above.. When CLK goes low, DQ changes to the next data bit and CLK goes high again to store the next bit. So in each data transmission CLK goes high and low 24 times for 3 bytes of data. The Nonin ipod has two output wires: one for TTL and the other for RS-232. The TTL wire is equivalent to the CLK bit shown in the timing diagram and the RS-232 bit is equivalent to the DQ bit.

\section{Respiration Rate}

The sensor for respiration rate is considerably more difficult to find. The Crossbow motes transmit data in pulse intervals approximately once every second. The data generated by sensors that read respiration rate will appear in a sinusoidal waveform. The frequency of the wave would have to be measured and analyzed on board and this data will be sent wirelessly. Currently we are unaware of the abilities of the MICA2's and MICA2DOT's onboard microcontroller, the Atmel ATMega 128. Assuming the microcontroller is capable of analyzing this data the sensors need only be connected to the ADC inputs. If the microcontroller is unable to process this data, we must find another microcontroller. As of now we have yet to find a suitable respiration rate sensor for our system.

Fig 11 shows the output of a pressure sensor used in a respiration rate device. The pressure inside the air bladder of the belt increases when the user inhales and decreases when the user exhales. The respiration rate is the frequency at which a person inhales and exhales within a time period, usually sixty seconds. The breath rate will vary throughout the day, whether the user is sleeping (respiration rate is very low) or exercising (respiration rate is higher). Certain precautions must be taken when implementing a system with an alarm that goes off based on breath rate since it varies so much. A respiration rate monitor will not be able to determine whether a person has fallen down the stairs and knocked unconscious or fallen asleep. Respiration rate monitors are normally used for physical training by working to maintain a person's heart and respiration rate while exercising.

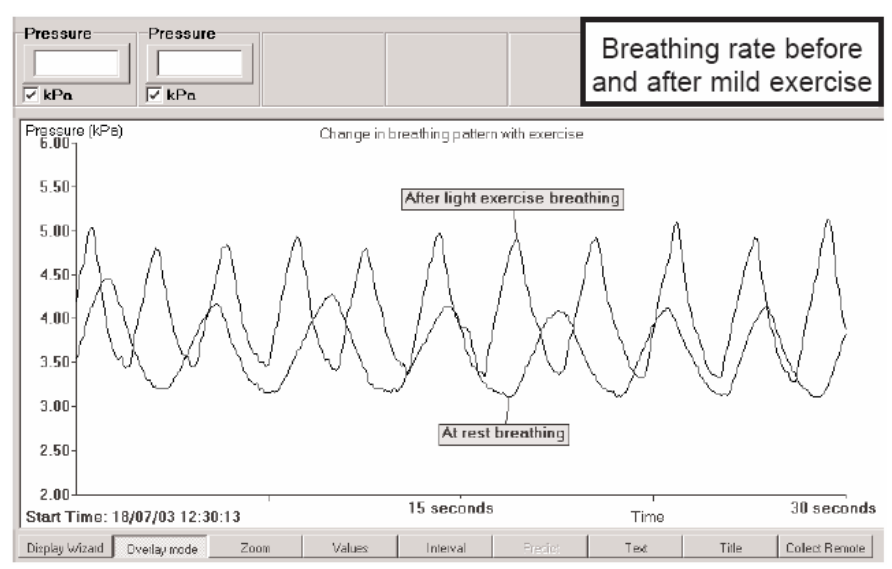

Fig 11: Breathing rate waveforms

There were several examples of respiration rate sensors found $[8,9]$. The first works by means of an inflatable belt which is strapped around the user's chest. The belt is then inflated and the expansion and contraction of the chest is analyzed with a pressure sensor. The pressure sensor analyzes the amount of pressure that increases and decreases as the user's chest squeezes the belt. The second example works in a similar manner: an elastic belt is strapped to the user's chest and the expansion and contraction of the belt is measured by (a unit which measures the expansion and contraction of an elastic belt). Fig 12 is an example of a respiration rate sensor.

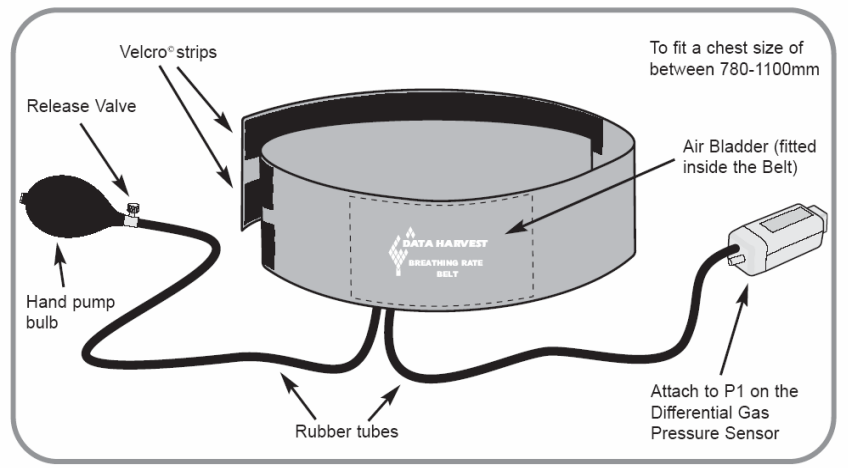

Fig. 12: An example of a respiration rate sensor. 
The tracking component of the Health Tracker 2000 will be handled by radio frequency identification tags (RFID). RFID systems transmit identity, in the form of a serial number, of a person or object wirelessly through radio waves. The system would consist of two main components: a reader and a tag [10]. If an RFID system is being used in a warehouse environment, a crate would have the tag inside and a person taking the inventory would carry a reader. The reader is used to point at the crate to obtain the tag's serial number which identifies the inventory.

In a passive RFID system the tag does not require any form of battery as power. The tag itself is powered by the radio frequency signal emitted by the reader. This provides a huge advantage in that the tag itself could be very tiny.

Integrating an RFID tracking ability to our system is very simple. We would simply add the reader to the base station and a tag to the unit worn by the user. Since the tags will be very small it wouldn't increase the overall size of the final system. The Health Tracker 2000 system does not have to be very accurate in tracking a patient because a receiver unit is placed in each room where the patient is located.

\section{Software}

When all sensors are connected to the mote, a program will be used to read the information and redirect the output to a database. From there another program that takes information from this database will analyze the information and send out warning signals.

In the final product, a graphical user interface (GUI) will need to be developed. The purpose of the GUI is for the base station program. This program will interpret the data and determine whether the users of Health Tracker 2000 are in critical condition. If so, an alert will show and a message will be sent through the Internet.

\section{Implementation}

This project is still ongoing. Thus far, we have integrated the following parts in our implementation: Crossbow MICA2 mote (MPR400CB), Crossbow MDA300CA Interface Board, National Semiconductors LM92 Precision Centigrade Thermal Sensor, and Nonin Integrated Pulse Oximetry Device (Ipod).

The thermal sensor was integrated into the MICA2 mote through the $\mathrm{I} 2 \mathrm{C}$ interface. The data read from the LM92 sensor is successfully sent to the base station wirelessly. The data was confirmed and displayed at the host computer. The wave in Fig 13 shows the temperature data recorded on the host computer in real-time.

We have encountered some interface problem when connecting the Ipod oximeter with the Crossbow motes. However, the device works when connected directly to a host computer. Figure 14 shows the blood oxygen concentration and heart rate of a user when the device clips onto a finger.

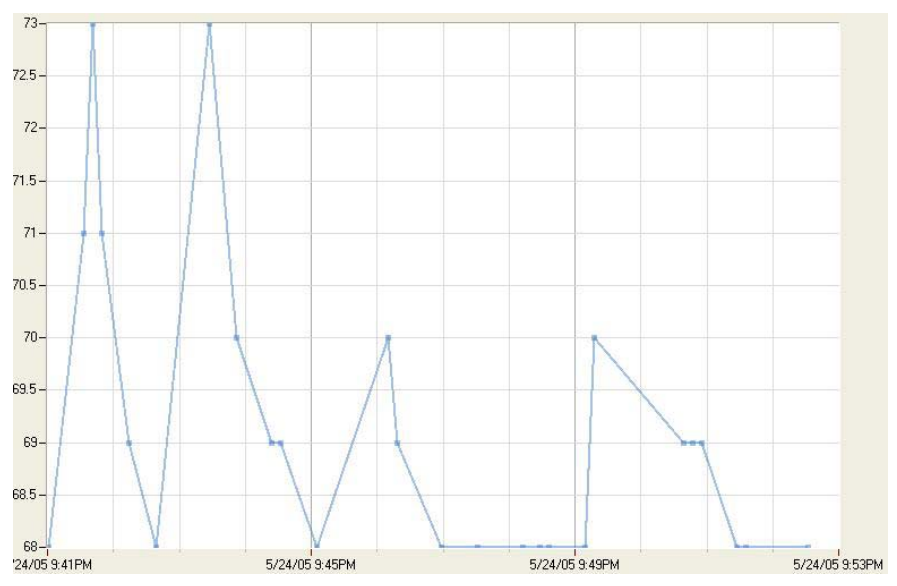

Fig. 13: Temperature data recorded and shown on the computer

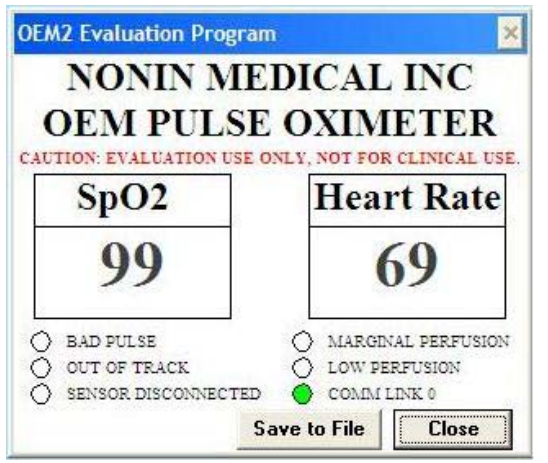

Fig. 14: Blood oxygen concentration and heart rate recorded and shown on the computer

\section{TESTING AND DISCUSSIONS}

Testing

The temperature data from the LM92 sensor was tested for correctness by connecting the serial data (SDA) channel to an oscilloscope. The room temperature was verified by comparing the LM92's reading with another thermometer. Once the data generated by the thermal sensor was verified, the temperature data received at the base station through wireless communication and our software interface is then verified. Some programming bugs, regarding the timing issue, in Crossbow Technology's system program library were fixed in this process.

\section{Discussion}

The vision of this project is to integrate various vital sign sensors with the "motes" provided by Crossbow Technology Inc. to provide a wireless health monitoring system. It is an ongoing project. Thus far, we have identified the temperature sensor, the oximetry sensor, the breath rate sensor and the RFID sensor as the key sensors in the system. At a first glance, the project seems trivial as only system integration is involved. However, as we proceed with the project, we found that different sensors have different data formats. System 
integration has to accommodate these different formats and different hardware ports. Thus far, we have only successfully integrated the temperature sensor and are still working on the other sensors. Upon complete integration of these sensors, a more research oriented issue is the establishment of an efficient sensor networks that can support large number of simultaneous users in a hospital or assisted living environment.

This project is supported by the Sensor Consortium in Stony Brook University and is funded by the National Science Foundation. The goal of the consortium is to promote and increase awareness of entrepreneurship and technology transfer activities on Long Island with a focus on national security and medical sensor systems. The team consists of undergraduate students Edward Teaw from Stony Brook University, Jason Farrell from Suffolk Community College, Amy Kesluk from Hofstra University, and Matthew Kane from SUNY Farmindale, graduate student Guofeng Hou from Stony Brook University, and faculty advisors Professor Wendy Tang, Professor Michael Gouzman from Stony Brook University. The undergraduate students each come from different schools, thus providing diverse educational backgrounds.

The main issue when dealing with a project with members from different schedules is communication. All four of the undergraduate members are full time students and employed as well. During the fall semester we were only able to meet at most once a week. A discussion group was created in Yahoo! Groups to be used as a discussion forum of new ideas and research findings. The service would automatically e-mail all members when a new message was posted. Creating this group helped maintain communication with all team members.

All members are required to take a course in Entrepreneurship at Stony Brook. Professor Gerrit Wolf provided information on how to build a business plan around a technical innovation. Several issues discussed were patents, timing of the product conception and release, and marketing the product.

We found such multidisciplinary team experience very challenging and yet rewarding. Aside from the technical issues, we also developed a business plan for our product, the Health Tracker 2000. On the technical side, thus far, we have integrated the temperature and the oximetry sensors with Crossbow's MICA2 motes. We plan to continue the development of this system by integrating other sensors such as the respiration rate sensor and the RFID sensors as described in Section II. Overall, we found the project provided us with an opportunity to build on our technical strength and expand our horizon to include entrepreneurial skills.

\section{CONCLUSIONS}

In this paper, we have described a Wireless Health Monitoring system that can wirelessly monitor vital signs of users in real-time and notify medical personnel and family members immediately in case of emergencies. With the aging of the US and the World population, the proposed system, the Health Tracker 2000, is expected to improve the health care system. The project is sponsored by the Sensor Consortium whose goal is to introduce to and increase awareness of entrepreneurial skills for engineering students.

\section{Acknowledgment}

This research is supported by the National Science Foundation under Grant No. EEC-0332605. Any opinions, findings and conclusions or recommendations expressed in this article are those of the authors and do not necessarily reflect the views of the National Science Foundation.

\section{REFERENCES}

[1] "History of Medicine." $\mathrm{http}: / /$ www.encyclopedia.com/html/section/medicine_historyofmedicine.a $\mathrm{sp}$

[2] "Health, United States, 2004" "http://www.cdc.gov/nchs/data/hus/hus04trend.pdf\#027" Sept. 2004

[3] Projected Population of the United States, by Age and Sex: 2000 to 2050" http://www.census.gov/ipc/www/usinterimproj/natprojtab02a.pdf" 01 Jul. 2004.

[4] "Home Digital Home: Wi-Fi Gets a Place At The Table" http://www.wirelessfidelitymag.com/articles/4c1 feat1.html. Dec. 2004.

[5] Choi, Jihong, John Han, Sunghoon Lee, Shantanu Tripathi. "Digi Trainer"

"http://kingkong.me.berkeley.edu/html/Me221/tradeshow_assets/trade_20 03/group_6_digi_trainer.htm" July 2004.

[6] Crossbow Technologies Inc., www.xbow.com.

[7] Nonin Medical Inc., www.nonin.com

[8] "PASPORT Respiration Rate Sensor" http://store.pasco.com/pascostore/showdetl.cfm?\&DID=9\&Product_ID $=5$ 3778\&Detail $=1$

[9] "Research on Sensing Human Affect" http://affect.media.mit.edu/areas.php?id=sensing

[10]Halfhill, Tom. "Is RFID Paranoia Rational?" Maximum PC. 14 Jan. 2005. 